\title{
Effect of posture on the intraocular pressure of patients with retinal vein obstruction
}

\author{
BILlie I. Williams and W. S. PEART \\ From the Western Ophthalmic Hospital, and Medical Unit, St Mary's Hospital, London
}

SUMMARY The effect of change of posture on the level of the intraocular pressure was studied in 22 patients who had suffered 'occlusion' of a central retinal vein, 14 patients with occlusion of a tributary of the central vein, and 23 normal subjects. The results showed a significant difference in behaviour between subjects in the control group and both groups of patients with retinal vein occlusions. In general it appears that the normal person maintains a relatively rigid control of ocular tension. The eyes are maintained at similar pressures which vary very little with change in posture. In contrast, the patient with a retinal vein occlusion (whether central or tributary) does not appear to have a similar degree of control. The eyes are not maintained at closely similar pressures, and each eye shows a change of pressure with change of posture which is considerably greater than that shown by the eyes of normal persons. The significance of these results is discussed with particular reference to the clinical association of retinal vein occlusion and chronic simple glaucoma, in which latter condition a similar lack of postural control of the intraocular pressure is found.

'Occlusion' of the central retinal vein or one of its tributaries is one of the commonest causes of sudden, serious and often permanent loss of vision seen in patients attending an eye clinic, and many of these patients are found to have a raised systemic arterial pressure. This condition must therefore be considered as a major complication of the retinopathy seen in these and other patients with hypertension of a comparable degree.

The cardinal feature of this retinopathy is an increase in the volume of the retinal veins. This is seen ophthalmoscopically as an increase in both their diameter and length, leading to the appearance of fat and tortuous vessels. Associated with this there is usually a variable degree of obscuration of the veins at the arteriovenous crossings due to an increase in the connective tissue sling which binds the artery and vein together at these points.

The appearance of the arteries is variable. They may be regular and give off a high reflex, or they may show irregularity of calibre and colour due to localised changes in their walls. When central vein occlusion supervenes, haemorrhages and exudates appear in all areas of the retina, and the veins appear dusky and grossly distended as though broken up into arched, ballooned segments which lie in the oedematous retina. When the vein involved

Address for reprints: Dr B. I. Williams, Medical Unit, St. Mary's Hospital, Praed Street, London W2 1NY is a tributary of the central vessel, a similar picture appears, with the difference that the changes are limited to the territory drained by the affected vessel and are virtually always situated distal to a point where it is crossed by an artery, fanning out to cover a wedge-shaped area with its apex at the arteriovenous crossing point.

The term venous 'occlusion' has come to replace venous 'thrombosis' since the studies of Verhoeff and others have shown that in the majority of these cases no true thrombus formation occurs (Verhoeff, 1907; Seitz, 1964). The term 'occlusion' may, however, also be a misnomer since retinal angiography with fluorescein has shown the affected vessel may be patent, when pictures are taken in the acute stage, as early as 1 day after the event (Smith et al., 1964). Perhaps the term retinal vein 'obstruction' is more appropriate, since we do not know whether the lumen of the affected vessel must necessarily be obliterated, if only momentarily, before the retinal changes characteristic of this condition appear.

Despite the common occurrence of both central and tributary vein obstruction and their clinical association with systemic hypertension, we have as yet no knowledge of the sequence of events which provokes these lesions in certain patients. In the broadest of terms it seemed to us that some abnormal variation in the level of pressure in the retinal veins must be taking place. Two observations, made on 
patients referred to the medical clinic at the Western Ophthalmic Hospital following retinal vein obstruction, seemed to offer support to this view and prompted the studies which will be described in this paper.

The observations were as follows: (1) Most affected patients became aware of their visual loss in the morning, usually quite soon after they had got out of bed; (2) very many of these patients noticed a diurnal variation in their vision, saying that they saw less well in the morning than in the afternoon or evening.

An analysis of the known diurnal variation of intraocular tension in normal persons has shown that, while the pattern of variation is not constant, most people $(65 \%)$ show maximum levels of tension at midnight or 5 a.m. (De Venecia and Davis, 1963.) Since our observations were noted in a similar proportion of patients it seemed possible that they might in some way be dependent on or at least related to these diurnal variations of pressure. Our hypothesis was that they reflected changes in the venous pressure occurring in the eye overnight and associated with leakage of fluid from capillaries, with resultant macular oedema. In the case of an established obstruction these events would be expected to cause a deterioration of vision in the morning with improvement as the day wears on and the sequence of pressure changes goes into reverse. By analogy a similar pattern of events could be supposed to be significant in the causation of an obstruction.

To test this hypothesis we decided to initiate a series of experiments designed to identify these supposed diurnal variations in the state of the retinal circulation. Our overall plan was to investigate a number of patients with various types of retinal vein obstruction by comparing morning and evening fluorescein angiograms of their retinae in the hope that we might identify such changes as alterations in venous calibre, capillary circulation, or degree of fluid exudation and be able to relate these to diurnal variations in visual acuity and systemic arterial and/or intraocular pressure.

During the course of preliminary experiments carried out on 6 of our patients with tributary vein obstructions we established that in all of them there was a diurnal variation in their intraocular tension, pressures recorded in the morning being consistently higher than those recorded in the evening in both eyes. The mean difference found in the affected eyes was $4.8 \mathrm{mmHg}$ and in the non-affected eyes $5.9 \mathrm{mmHg}$.

Since we had been trying to mimic the normally occurring situation, morning readings had been taken while the patient was still in bed, in the supine position, whereas evening readings were taken with the patient in the sitting position, a Perkins handheld applanation tonometer being used to make the measurements. This difference in posture could not, of course, be presumed as the sole cause of the different levels of pressure found, since the diurnal variation for any patient has been established using the Schiøtz tonometer, which involves the patient's being in the same position for all readings, that is, supine or semisupine. Nevertheless, we were interested to know how significant posture was, and so we decided to investigate the effect of relatively immediate changes of posture per se on levels of systemic arterial and intraocular pressure in certain of our patients and compare the results with those found in normal persons.

\section{Patients and methods}

\section{SUBJECTS}

We examined 3 groups of subjects, all of whom received a preliminary clinical examination: (1) 22 cases of central vein obstruction; (2) 14 cases of tributary vein obstruction; (3) 23 control subjects with no evidence of ocular disease.

The cases of central and tributary retinal vein obstruction were selected irrespective of age, sex, the supposed aetiology of the obstruction, or the presence of systemic hypertension. The duration of the obstruction varied among the subjects, and some were receiving coincident systemic therapy with drugs such as hypotensives, diuretics, steroids, etc., at the time of the investigation. One case of central vein obstruction was receiving local treatment (pilocarpine drops $1 \%$ twice daily) for chronic simple glaucoma which had developed after the obstruction.

\section{AGE AND SEX}

(1) Central vein obstruction. This group contained 12 male and 10 female patients. Their ages ranged from 26 years to 73 years, the mean age for the group being $59 \cdot 4$ years.

(2) Tributary vein obstruction. This group contained 8 male and 6 female patients. Their ages ranged from 52 years to 70 years, the mean age for the group being 60.8 years.

(3) Control subjects. This group contained 5 male and 18 female patients. Their ages ranged from 19 years to 70 years, the mean age for this group being $45 \cdot 8$ years.

\section{METHOD}

The subject was asked to sit quietly near one end of an examination couch for 15 minutes. The arterial pressure was then measured with a random recording sphygmomanometer, the average of 3 


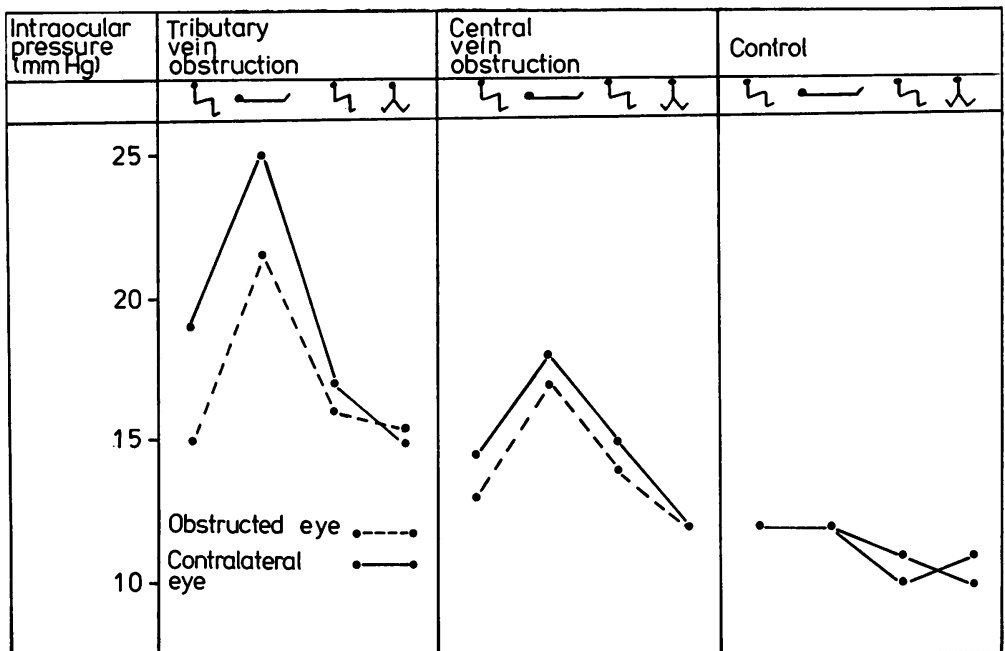

Fig. 1 Typical postural pressure responses of 3 subjects in the age group 50 to 60 years readings being recorded. Oxybuprocaine $0.3 \%$ drops and fluorescein $0.125 \%$ were then instilled in both conjunctival sacs and the intraocular pressure measured with a Perkins hand-held applanation tonometer. The right eye was always examined first, 3 readings being taken, the mean of which was recorded. The subject was then asked to lie down (without getting up meanwhile), and after 15 minutes the arterial and intraocular pressures were measured and recorded as before. The subject was then asked to sit up, and after a further 15 minutes the readings were once more taken. Final measurements were taken with the patient in the standing position, these being recorded after he had been walking about at his normal pace for 15 minutes in the outpatient department.

We thus had 4 consecutive sets of readings for the various body positions, namely, sitting, supine, sitting, standing.

\section{Results}

\section{INTRAOCULAR PRESSURE}

An analysis of our results was made with respect to the following points: The relative levels of tensions in the 2 eyes, measured with the subject in the original sitting position; the difference in intraocular pressure existing between the 2 eyes of any subject, as measured in the original sitting position; the magnitude of any change in ocular pressure occurring when the subject went from the original sitting position to one in which he was lying down; the difference between the maximum and minimum intraocular pressures recorded in any eye, irrespective of the posture adopted (maximum amplitude).

The first measurements we considered were the levels of pressure in the obstructed and contralateral eye while the patient was in the original sitting position. We found that the pressure of the obstructed eye was lower than that of its colleague in $75 \%$ of those cases with tributary vein obstruction and in $68 \%$ of those cases with a central obstruction. The pressures were as follows (mean \pm SD):

$\begin{array}{lll} & \text { Obstructed eye } & \text { Contralateral eye } \\ \text { Central vein obstruction } & 15.3 \mathrm{~mm} \pm 2.7 & 17.0 \mathrm{~mm} \pm \mathbf{3 . 2} \\ \text { Tributary vein obstruction } & 14.7 \mathrm{~mm} \pm 2.3 & 15.8 \mathrm{~mm} \pm \mathbf{2 . 3}\end{array}$

In patients with central vein obstruction the levels of intraocular pressure in the obstructed eyes were significantly below those of their contralateral pairs (paired $t=3.29,2 \mathrm{P}<0.005$ ), whereas the pressures in the 2 eyes did not differ significantly in patients with tributary vein obstruction (paired $t=1 \cdot 27$, $2 \mathrm{P}<0.3)$. These findings are in agreement with those described by J. Foster Moore (1924) in his classic monograph.

We then considered the magnitude of any difference in pressure existing between the obstructed and contralateral eyes and compared this with that existing between the right and left eyes of the control subjects. Reference to Table 1 shows that the group of control subjects had a mean difference of $1.0 \pm$ $1.0 \mathrm{mmHg}$, while the group of patients with tributary vein obstruction had a mean difference of $2 \cdot 6 \pm$ $1.9 \mathrm{mmHg}$, which is significantly greater (unpaired $t=2.9,2 \mathrm{P}<0.01)$. The group of patients with central vein obstruction had a mean difference of $2 \cdot 4 \pm 2 \cdot 1 \mathrm{mmHg}$, which is also significantly greater than that found in the control group (unpaired $t=2 \cdot 86,2 \mathrm{P}<0.01$ ).

Change of position from sitting to lying also showed a further difference between patients and control subjects. The control subjects had a mean 
Table 1 Differences in intraocular pressure between subjects in different groups and between eyes within groups

\begin{tabular}{|c|c|c|c|c|c|c|}
\hline \multirow{3}{*}{ Groups of subjects } & \multirow{3}{*}{ No. } & \multicolumn{5}{|c|}{ Intraocular pressure $(\mathrm{mmHg})$} \\
\hline & & \multirow[t]{2}{*}{$\begin{array}{l}\text { Mean difference } \\
\text { right/left eyes }\end{array}$} & \multicolumn{2}{|c|}{$\begin{array}{l}\text { Mean rise on changing from } \\
\text { sitting to lying }\end{array}$} & \multicolumn{2}{|c|}{ Mean maximum amplitude } \\
\hline & & & Obstructed eye & Fellow eye & Obstructed eye & Fellow eye \\
\hline $\begin{array}{r}\text { Tributary vein } \\
\text { obstructions }\end{array}$ & 14 & $2 \cdot 6 \pm 1 \cdot 9$ & $3 \cdot 1 \pm 2 \cdot 3$ & $2 \cdot 1 \pm 2 \cdot 2$ & $4 \cdot 8+2 \cdot 5$ & $4 \cdot 6 \pm 2 \cdot 5$ \\
\hline \multirow{2}{*}{$\begin{array}{l}\text { Central vein } \\
\text { obstructions }\end{array}$} & 22 & $2 \cdot 4 \pm 2 \cdot 1$ & $1 \cdot 8 \pm 2 \cdot 4$ & $2 \cdot 6 \pm 2 \cdot 3$ & $4 \cdot 6 \pm 1.9$ & $5 \cdot 1 \pm \cdot 2 \cdot 8$ \\
\hline & & & \multicolumn{2}{|l|}{ Mean of 46 eyes } & \multicolumn{2}{|l|}{ Mean of 46 eyes } \\
\hline Control subjects & 23 & $1 \cdot 0 \pm 1 \cdot 0$ & $0 \cdot 6 \pm 1 \cdot 2$ & & $2 \cdot 3 \pm 1 \cdot 6$ & \\
\hline
\end{tabular}

rise of $0.6 \pm 1 \cdot 2 \mathrm{mmHg}$ for the 46 eyes in this group, which was compared separately with that found in each of the 2 eyes of patients in the other 2 groups. In the group of patients with tributary vein obstruction the mean rise was significantly greater in both the obstructed and contralateral eyes than that found in the control eyes. The mean rise in the obstructed eyes was $3 \cdot 1 \pm 2.3 \mathrm{mmHg}$ (unpaired $t=$ $3.85,2 \mathrm{P}<0.001)$ and in the contralateral eyes $2 \cdot 1 \pm 2 \cdot 2 \mathrm{mmHg}$ (unpaired $t=2 \cdot 42,2 \mathrm{P}<0.02$ ). In the group with central vein obstruction the mean rise was also significantly greater in both the obstructed and contralateral eyes than that found in the control eyes. The mean rise in the obstructed eyes was $1.8 \pm 2.4 \mathrm{mmHg}$ (unpaired $t=2 \cdot 22$, $2 \mathrm{P}<0.05)$ and $2.6 \pm 2.3 \mathrm{mmHg}$ in the contralateral eyes (unpaired $t=3.45,2 \mathrm{P}<0.001$ ).

The final quantity which we considered was the mean of the maximum amplitude. The control subjects showed a mean amplitude of $2 \cdot 3 \pm$ $1.6 \mathrm{mmHg}$ for the 46 eyes which we compared separately with each of the 2 eyes of patients in the other groups. In the group of patients with tributary vein obstruction the mean maximum amplitude was significantly greater in both the obstructed and contralateral eyes than that found in the control eyes. The mean in the obstructed eyes was $4.8 \pm 2.5$ $\mathrm{mmHg}$ (unpaired $t=3.52,2 \mathrm{P}<0.001$ ) and in the contralateral eyes, $4.6 \pm 2.5 \mathrm{mmHg}$ (unpaired $t=$ $3.24,2 \mathrm{P}<0.005$ ). In the group with central vein obstruction, the mean maximum amplitude was also significantly greater in both the obstructed and contralateral eyes than that found in the control eyes. The mean maximum amplitude was $4.6 \pm$ $1.9 \mathrm{mmHg}$ in the obstructed eyes (unpaired $t=$ $4.89,2 \mathrm{P}<0.001)$ and $5.1 \pm 2.8 \mathrm{mmHg}$ in the contralateral eyes (unpaired $t=4.31,2 \mathrm{P}<0.001$ ).

SYSTEMIC ARTERIAL PRESSURE

The arterial pressures (mean \pm SD) recorded with the patient in the initial sitting position for the 3 groups of subjects were found to be as follows: (1) Patients with central vein obstruction, $149( \pm 31)$ / $89( \pm 14)$. (2) Patients with tributary vein obstruction, $151( \pm 22) / 98( \pm 12)$. (3) Normal subjects, 117 $( \pm 18) / 74( \pm 11)$.

Many of the patients in the first 2 groups, however, were receiving hypotensive drugs at the time of the study. The mean arterial pressures for these 2 groups as recorded in their outpatient notes before the commencement of hypotensive therapy were as follows: (1) Central vein obstruction, $157( \pm 34) / 93( \pm 14)$. (2) Tributary vein obstruction, $169( \pm 20) / 103( \pm 14)$.

It is seen from these figures that the patients who suffered a retinal vein obstruction had a higher mean systemic pressure than the persons in the control group and that those with a tributary obstruction had a somewhat higher mean pressure than those with a central obstruction at the time of the experiment. The level of hypertension even before treatment was, however, modest in the majority of patients, and some patients were normotensive.

We found no correlation between variations in systemic and intraocular pressure associated with the same change in posture (Hetland-Eriksen, 1966).

\section{COINCIDENT THERAPY}

At the time the experiments were carried out some of the patients with venous obstruction were receiving various systemic drugs including propranolol, methyldopa, clonidine, hydrochlorothiazide, Modurectic (amiloride and hydrochlorothiazide), bendrofluazide, cyclopenthazide, frusemide, spironolcatone, quinine sulphate, amitriptyline, allopurinol, nitrazepam, cholordiazepoxide, indomethacin, and prednisone. Fifteen out of 36 patients $(41.6 \%)$ were receiving no treatment, while 21 patients $(58.4 \%)$ were receiving 1 or more of the drugs listed above. We were unable to detect any significant difference in the pattern of the intraocular pressure response to change of posture between 
those patients receiving any of the drugs and those who were not.

\section{DURATION OF OBSTRUCTION}

The duration of the obstruction varied between 1 month and 5 years. The length of time between onset of obstruction and our measurements varied between 1 month and 3 years in tributary vein obstructions and between 6 weeks and 18 months in central vein obstructions. Although no obvious relationship is apparent between the duration of the obstructions and the pattern of the intraocular pressure response, we intend to repeat our observations at a suitable interval in order to assess any change in individual patients.

\section{Discussion}

From the results above it is seen that persons in our control group behave differently from the groups of patients with retinal vein obstruction. In general it appears that the normal subject maintains a relatively rigid control of ocular tension. The eyes are maintained at similar pressures which vary very little with change in posture. In contrast the patient with a retinal vein obstruction (whether central or tributary) does not appear to have a similar degree of control. The eyes are not maintained at closely similar pressures, and each eye shows a change of pressure with change of posture. Typically these eyes show a rise of about 2 to $3 \mathrm{mmHg}$ associated with the change from sitting to lying (that is, approximately 4 to 6 times the change shown by normal eyes) and a maximum amplitude of pressure change between 4.5 and $5 \mathrm{mmHg}$ (that is, more than twice that of normal eyes).

We have not been able so far to detect any variation in the intraocular pressure response dependent on levels of systemic pressure (or their variation with posture), the absence or presence of any therapy, or the duration of the obstruction, although we intend to study this further.

Two points of interest arise from these results. Firstly, the fact that both eyes-that is, obstructed and contralateral-show similar responses raises the question whether we have been measuring a quantity which is dependent on an abnormality which was present in both eyes before the obstruction occurred or is the result of the fact that 1 eye became the seat of an obstruction.

Secondly, various workers have shown that eyes with chronic simple glaucoma show a similar response to postural changes (Hetland-Ericksen, 1966; Anderson and Grant, 1973; Krieglstein and Langham, 1975; Langham, 1975). For the most part their studies have been concerned with the behaviour of eyes with open-angle, chronic simple glaucoma as compared with normal eyes, and they have reported a consistently different response in these 2 groups. The essential difference found has been related to the effect of changing the subject's position from sitting to lying. Normal persons have been recorded as showing lower rises of pressure following this manoeuvre. For example, Krieglstein and Langham (1975) studied 77 normal and 65 glaucomatous eyes and found an average rise of $2.9 \mathrm{mmHg}$ in normal eyes as compared with $3.9 \mathrm{mmHg}$ in glaucomatous eyes.

The similarity between the response shown by these glaucomatous patients and that found in our group of patients is interesting in view of the wellestablished clinical association between retinal vein obstruction and chronic simple glaucoma. The aetiological relationship that exists between these two conditions, however, remains obscure.

A number of clinical studies of the coincidence of the two conditions in 1 patient suggests that primary glaucoma has no special relationship with tributary vein obstruction but a highly significant one with central vein obstruction (Moore, 1924; Vannas and Tarkkanen, 1960; Bertelsen, 1961).

Thus Vannas and Tarkkanen (1960) found evidence of simple glaucoma as a pre-existing condition in only $10 \%$ of their patients with tributary vein obstructions, an incidence no greater than that found in a group of persons of comparable age selected from the general population, while they found an incidence of $42 \%$ in their cases presenting with a central obstruction. Other workers have found values for the coincidence of primary glaucoma and central vein obstruction as follows: $11 \%$ Baendstrup (1952); $43 \%$ Bertelsen (1961); $35 \%$ Raitta (1965).

Since these 2 groups of eyes-namely, glaucomatous and obstructed-appear to suffer from an inability to control their pressure in response to change of body position, our results might appear to suggest that eyes which develop chronic simple glaucoma and eyes which develop vein obstruction share a common defect which can produce either disease or both, depending on other as yet unknown factors. We have been impressed, however, with the similarity of the response in both tributary and central vein obstructions, although the mean rise of pressure on adoption of the supine position as well as the mean of the maximum amplitude was somewhat greater in the contralateral eye in those cases with a central obstruction. This may be of interest in view of the results of tonographic studies made by Vannas and Takkanen (1960) and Bertelsen (1961) on both eyes of patients with central vein obstruction. These studies showed that there was a 
greater facility of outflow in the obstructed as compared with the contralateral eyes.

Also of interest is the work of Inglima (1966), who performed postural tests on patients with unilateral simple glaucoma and found an abnormal response in the glaucomatous eyes but not in the contralateral normal eyes (Inglima, 1966). He carried out tonography on groups of normal and glaucomatous eyes and found a statistically significant relationship between facility of outflow and rise of intraocular pressure associated with the patient's change from a sitting to a supine position, that is, the lower the outflow the greater the rise of pressure. The postural effect was present in both eyes when both eyes suffered from an impaired facility of outflow and not otherwise.

Further studies are required to evaluate the significance of our findings for the understanding of the pathogenesis of retinal vein obstruction.

We gratefully acknowledge the support given by the Joint Standing Research Committee, St. Mary's Hospital, London, W2, and the Wolfson Foundation.

\section{References}

Anderson, D. R., and Grant, W. M. (1973). The influence of position on intraocular pressure. Investigative Ophthalmology, 12, 204-212.

Braendstrup, P. (1952). Centralvenethrombose-primaert glaucom. Nordisk Medicin, 48, 1668-1669.
Bertelsen, T. I. (1961). The relationship between thrombosis in the retinal veins and primary glaucoma. Acta Ophthalmologica, 39, 603-613.

De Venecia, G., and Davis, M. D. (1963). Diurnal variation of intraocular pressure in the normal eye. Archives of Ophthalmology, 69, 752-759.

Hetland-Eriksen, J. (1966). On tonometry, 5. The pressure of glaucomatous eyes measured in the sitting and the lying positions by means of the Goldmann applanation tonometer. Acta Ophthalmologica, 44, 515-521.

Inglima, R. (1966). Effect of patient position on applanometer readings. Eye, Ear, Nose and Throat Monthly, 45, 64-69.

Krieglstein, G. K., and Langham, M. E. (1975). Influence of body position on the intraocular pressure of normal and glaucomatous eyes. Ophthalmologica, 171, 135-145.

Langham, M. E. (1975). Vascular pathophysiology of the ocular postural response. A pneumotonometric study. Transactions of the Ophthalmological Societies of the United Kingdom, 95, 281-287.

Moore, J. F. (1924). Retinal Venous Thrombosis. British Journal of Ophthalmology, Monograph Supplement, 2.

Raitta, C. (1965). Der Zentralvenen- und Netzhautvenenverschluss. Acta Ophthalmologica, Supplementum 83, 123.

Vannas, S., and Tarkkanen, A. (1960). Retinal vein occlusion and glaucoma. Tonographic study of the incidence of glaucoma and its prognistic significance. British Journal of Ophthalmology, 44, 583-589.

Seitz, R. (1964). The Retinal Vessels, translated by F. S. Blodie. Mosby: St. Louis.

Smith, V., Paton, A., and Rubenstein, K. (1964). Arterial insufficiency in retinal vein occlusion.Transactions of the Ophthalmological Societies of the United Kingdom, 84, 559-595.

Verhoeff, F. H. (1907). Obstruction of the central retinal vein. Archives of Ophthalmology, 36, 1-36. 